\title{
Binge Drinking Among Adults, by Select Characteristics and State - United States, 2018
}

\author{
Michele K. Bohm, MPH${ }^{1}$; Yong Liu, MD ${ }^{1}$; Marissa B. Esser, PhD ${ }^{1}$; Jessica B. Mesnick, MPH${ }^{1}$; Hua Lu, MS ${ }^{1}$; Yi Pan, PhD²; Kurt J. Greenlund, PhD ${ }^{1}$
}

Excessive alcohol use* is associated with disease, injury, and poor pregnancy outcomes and is responsible for approximately 95,000 deaths in the United States each year (1). Binge drinking (five or more drinks on at least one occasion for men or four or more drinks for women) is the most common and costly pattern of excessive alcohol use (2). CDC analyzed data from the 2018 Behavioral Risk Factor Surveillance System (BRFSS) to estimate past 30-day binge drinking prevalence, frequency, and intensity (number of drinks per occasion), overall and by select characteristics and state. The overall unadjusted prevalence of binge drinking during the past 30 days was 16.6\%, representing an estimated 38.5 million U.S. adults aged $\geq 18$ years; prevalence was highest $(26.0 \%)$ among those aged 25-34 years. The age-standardized binge drinking prevalence was higher among men (22.5\%) than among women (12.6\%), increased with income, and was highest among non-Hispanic White adults and adults in the Midwest Census region. Statelevel age-standardized binge drinking prevalence ranged from $10.5 \%$ (Utah) to $25.8 \%$ (Wisconsin). Among adults who reported binge drinking, $25.0 \%$ did so at least weekly, on average, and $25.0 \%$ consumed at least eight drinks on an occasion. To reduce binge drinking, the Community Preventive Services Task Force recommends increasing alcohol taxes and implementing strategies that strengthen regulations to reduce alcohol availability. ${ }^{\dagger}$ The U.S. Preventive Services Task Force recommends clinicians screen adults for alcohol misuse in primary care settings and provide counseling as needed. $\$$

BRFSS is an ongoing, state-based, random-digit-dialed, landline and cellular telephone survey of the U.S. noninstitutionalized adult population that collects health-related data nationwide.9 In 2018, the median survey response rate $^{* *}$ for all states and the District of Columbia was $49.9 \%$ (range $=38.8 \%-67.2 \%) .{ }^{\dagger \dagger}$ CDC analyzed data from 398,485

\footnotetext{
* Excessive alcohol use includes binge drinking, heavy drinking (i.e., 15 or more drinks per week for men; eight or more drinks per week for women), and any drinking by pregnant women or persons aged $<21$ years. https://www.cdc. gov/alcohol/fact-sheets/alcohol-use.htm

$\dagger$ https://www.thecommunityguide.org/topic/excessive-alcohol-consumption

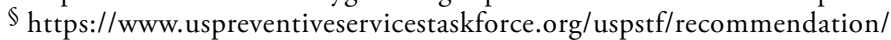
unhealthy-alcohol-use-in-adolescents-and-adults-screening-and-behavioralcounseling-interventions

' https://www.cdc.gov/brfss/annual_data/2018/pdf/overview-2018-508.pdf

** Response rates for BRFSS are calculated using standards set by the American Association for Public Opinion Research. https://www.aapor.org/StandardsEthics/Standard-Definitions-(1).aspx

$\dagger \dagger$ https://www.cdc.gov/brfss/annual_data/2018/pdf/2018-sdqr-508.pdf
}

respondents aged $\geq 18$ years in the 2018 BRFSS to estimate past 30-day binge drinking prevalence, frequency, and intensity. Binge drinking prevalence and frequency were assessed with the question, "Considering all types of alcoholic beverages, how many times during the past 30 days did you have 5 ( 4 for women) or more drinks on an occasion?” \$\$ Intensity was assessed with the question, "During the past 30 days, what is the largest number of drinks you had on any occasion?" (3). Unadjusted and age-standardized (to the 2000 U.S. standard population) binge drinking prevalence and $95 \%$ confidence intervals (CIs) were estimated overall. Age-standardized prevalence was also estimated by respondents' sociodemographic characteristics (except prevalence by age group), including sex, race/ethnicity, income, marital status, veteran status, education, region, county urbanization level, 99 and state. State-level prevalence estimates and 95\% CIs were grouped into tertiles to identify geographic patterns. Because of the highly right-skewed distribution of the data, similar measures of binge drinking frequency and intensity among adults reporting binge drinking were estimated with medians and variances derived using Taylor series linearization. The means and 75 th and 90 th percentiles for frequency and intensity were also calculated to further characterize the distributions of these measures. Statistically significant differences between medians were defined as $\mathrm{p}<0.05$ using pairwise tests and nonoverlapping CIs. All analyses were performed using SAS-callable SUDAAN (version 11.0.3; RTI International), and sampling weights were applied to account for complex sampling design, including nonresponse bias and noncoverage errors, and to improve representation of the adult U.S. population in different states.

In 2018, the overall nationwide unadjusted binge drinking prevalence among U.S. adults was $16.6 \%$ $(95 \% \mathrm{CI}=16.3 \%-16.8 \%)$, representing an estimated 38.5 million adults (Table 1); prevalence was highest among adults aged 25-34 years (26.0\%). Age-standardized binge drinking prevalence was $17.4 \%(95 \% \mathrm{CI}=17.2 \%-17.7 \%)$ and varied by sociodemographic group and by state (range $=10.5 \%$ [Utah] to 25.8\% [Wisconsin]) (Figure) (Supplementary Table, https://stacks.cdc.gov/view/cdc/110373). Binge drinking prevalence was significantly higher among men $(22.5 \%)$ than

\footnotetext{
$\mathbb{\$ S}$ https://www.cdc.gov/brfss/questionnaires/pdf-ques/2018_BRFSS_English_ Questionnaire.pdf

99 https://www.cdc.gov/nchs/data_access/urban_rural.htm
} 
TABLE 1. Prevalence of binge drinking among adults aged $\geq 18$ years, by selected characteristics - Behavioral Risk Factor Surveillance System, United States, ${ }^{*} 2018$

\begin{tabular}{|c|c|c|}
\hline Characteristic & $\begin{array}{l}\text { Weighted no. }{ }^{\dagger} \text { of } \\
\text { adults reporting } \\
\text { binge drinking, } \\
\text { x } 1,000\end{array}$ & $\begin{array}{c}\text { Binge drinking } \\
\text { prevalence, } \\
\%(95 \% \mathrm{Cl})\end{array}$ \\
\hline Overall, unadjusted & 38,544 & $16.6(16.3-16.8)$ \\
\hline Overall, age-adjusted & NA & $17.4(17.2-17.7)$ \\
\hline \multicolumn{3}{|l|}{ Age group, yrs } \\
\hline $18-24$ & 7,010 & $24.0(23.1-24.9)$ \\
\hline $25-34$ & 10,595 & $26.0(25.3-26.7)$ \\
\hline $35-44$ & 7,579 & $20.4(19.7-21.0)$ \\
\hline $45-64$ & 10,871 & $14.3(13.9-14.7)$ \\
\hline$\geq 65$ & 2,489 & $5.0(4.8-5.3)$ \\
\hline \multicolumn{3}{|l|}{ Sex } \\
\hline Men & 24,603 & $22.5(22.0-22.9)$ \\
\hline Women & 13,941 & $12.6(12.2-12.9)$ \\
\hline \multicolumn{3}{|l|}{ Race/Ethnicity } \\
\hline White, non-Hispanic & 25,654 & $19.7(19.4-20.0)$ \\
\hline Black, non-Hispanic & 3,570 & $13.4(12.7-14.2)$ \\
\hline Hispanic & 6,483 & $16.3(15.5-17.2)$ \\
\hline Al/AN, non-Hispanic & 336 & $16.1(14.0-18.6)$ \\
\hline A/PI, non-Hispanic & 1,281 & $9.7(8.7-10.8)$ \\
\hline Other, non-Hispanic & 738 & $16.9(15.6-18.2)$ \\
\hline \multicolumn{3}{|l|}{ Annual household income } \\
\hline$<\$ 25,000$ & 7,072 & $14.6(14.1-15.2)$ \\
\hline$\$ 25,000-\$ 49,999$ & 7,545 & $17.8(17.1-18.4)$ \\
\hline$\$ 50,000-\$ 74,999$ & 5,519 & $19.0(18.3-19.8)$ \\
\hline$\geq \$ 75,000$ & 14,761 & $21.4(20.9-21.9)$ \\
\hline \multicolumn{3}{|l|}{ Marital status } \\
\hline Married $^{* *}$ & 19,318 & $16.6(16.2-17.0)$ \\
\hline Divorced/Separated/Widowed & 5,820 & $19.4(18.2-20.7)$ \\
\hline Never married & 13,243 & $18.5(17.9-19.1)$ \\
\hline \multicolumn{3}{|l|}{ Veteran status } \\
\hline Veteran & 4,015 & $20.9(19.8-21.9)$ \\
\hline Nonveteran & 34,494 & $17.1(16.8-17.4)$ \\
\hline
\end{tabular}

among women (12.6\%) and was highest among non-Hispanic White adults (19.7\%), those with annual household incomes $\geq \$ 75,000$ (21.4\%), those who were never married (18.5\%) or were divorced/separated/widowed (19.4\%), and veterans $(20.9 \%)$. Binge drinking prevalence was significantly higher among adults with a college degree (18.9\%) than among adults with less than a high school diploma (14.9\%). States with higher binge drinking prevalences clustered in the Midwest and Northeast.

Among adults who reported binge drinking, the median frequency was 1.7 (mean $=4.6)$ binge drinking occasions during the past 30 days, and the median intensity was $5.5($ mean $=7.2)$ drinks on an occasion. (Table 2). The upper frequency quartile was $>4.0(95 \% \mathrm{CI}=3.9-4.1)$ binge drinking occasions in the past 30 days and the upper intensity quartile was $>7.7$ (95\% CI $=7.6-7.8)$ drinks on an occasion. Median binge drinking frequency and intensity were significantly higher among men (1.9 occasions and 5.9 drinks, respectively) than among women ( 1.4 occasions and 4.5 drinks, respectively), and decreased with education level. Median binge drinking
TABLE 1. (Continued) Prevalence of binge drinking among adults aged $\geq 18$ years, by selected characteristics - Behavioral Risk Factor Surveillance System, United States, ${ }^{*} 2018$

\begin{tabular}{lcc}
\hline Characteristic & $\begin{array}{c}\text { Weighted no. }{ }^{\dagger} \text { of } \\
\text { adults reporting } \\
\text { binge drinking, } \\
\mathbf{x} 1,000\end{array}$ & $\begin{array}{c}\text { Binge drinking } \\
\text { prevalence, } \\
\%\end{array}$ \\
\hline Education level & $(95 \% \mathrm{Cl})$ \\
\hline $\begin{array}{l}\text { Less than high school graduate } \\
\text { High school graduate or equivalent }\end{array}$ & 4,116 & $14.9(14.0-15.8)$ \\
Some college & 10,363 & $17.2(16.7-17.8)$ \\
College graduate & 12,862 & $18.4(17.9-18.9)$ \\
U.S. Census region ${ }^{\dagger \dagger}$ & 11,154 & $18.9(18.4-19.3)$ \\
Northeast & & \\
Midwest & 6,742 & $17.9(17.3-18.5)$ \\
South & 9,340 & $20.0(19.6-20.5)$ \\
West & 13,688 & $16.3(15.8-16.8)$ \\
County urban-rural status ${ }^{\S}$ & 8,775 & $16.6(16.1-17.1)$ \\
Large central metro & & \\
Large fringe metro & 12,298 & $17.7(17.1-18.2)$ \\
Medium metro & 9,457 & $17.3(16.8-17.9)$ \\
Small metro & 7,979 & $17.5(17.0-18.0)$ \\
Micropolitan & 3,519 & $17.7(17.1-18.4)$ \\
Noncore & 3,083 & $16.7(16.1-17.4)$ \\
\hline Abbreviations: & 2,210 & $16.5(15.7-17.3)$ \\
\hline
\end{tabular}

Abbreviations: $\mathrm{A} / \mathrm{PI}=$ Asian/Pacific Islander; $\mathrm{Al} / \mathrm{AN}=$ American Indian/Alaska Native; $\mathrm{Cl}=$ confidence interval; $\mathrm{NA}=$ not applicable.

* The study sample included 398,485 adult respondents aged $\geq 18$ years from all 50 states and the District of Columbia with complete information on age, sex, and binge drinking. Weighted numbers were derived through application of survey weights.

${ }^{\dagger}$ Categories in subgroups might not sum to total because of missing responses for some variables.

$\S$ Binge drinking was defined as consuming five or more drinks (men) or four or more drinks (women) on at least one occasion during the past 30 days.

I Prevalence estimates for all characteristics except age group were agestandardized to the 2000 U.S. standard population.

** Married includes unmarried cohabitating couples.

${ }^{t+}$ Regions were based on U.S. Census Bureau definitions. https://www2.census. gov/geo/pdfs/maps-data/maps/reference/us_regdiv.pdf

$\S \S$ Counties were classified using the 2013 National Center for Health Statistics Urban-Rural Classification Scheme for Counties. https://www.cdc.gov/nchs/ data/series/sr_02/sr02_166.pdf

intensity was highest among adults aged 18-24 years and decreased with age. Median binge drinking frequency among states ranged from 1.5 occasions (eight states) to 2.1 occasions (Mississippi) in the past 30 days; median binge-drinking intensity on an occasion ranged from 5.2 drinks (New Jersey, District of Columbia, and Connecticut) to 6.4 drinks (West Virginia).

\section{Discussion}

During 2018, one in six U.S. adults reported binge drinking during the past 30 days, increasing their risk for many preventable adverse health outcomes. Among those who binge drank, one half did so at least twice per month; one half of men consumed at least six drinks and one half of women consumed at least five drinks on a binge occasion. These median values are lower than the mean values for binge drinking frequency and intensity, but better represent how often adults who binge drink typically do so and how many drinks they usually consume. 
FIGURE. Prevalence of binge drinking* among adults aged $\geq 18$ years - Behavioral Risk Factor Surveillance System, United States, ${ }^{\dagger} 2018$

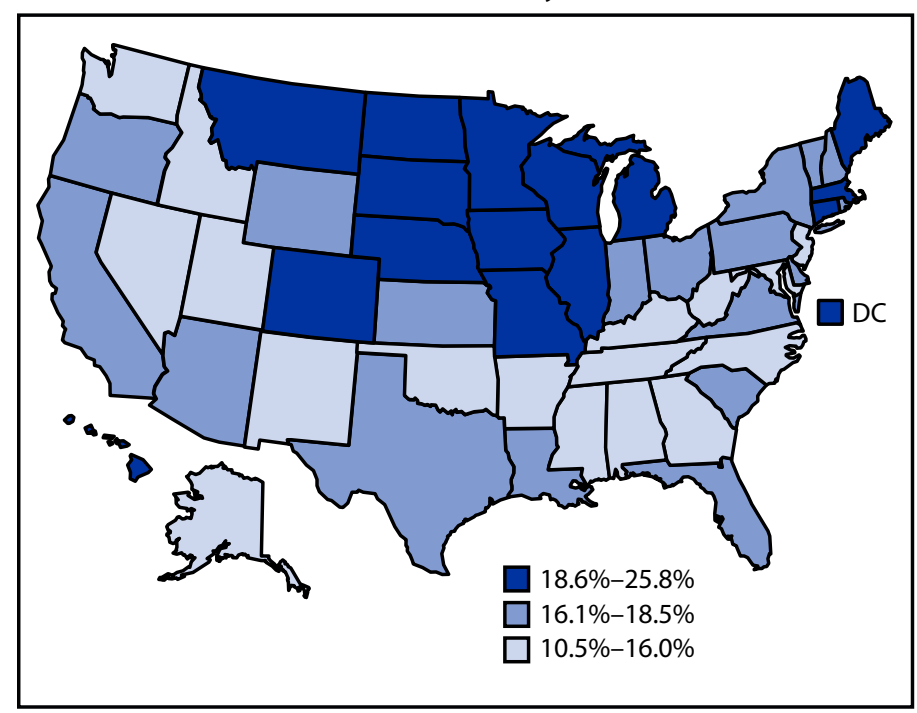

Abbreviation: $\mathrm{DC}=$ District of Columbia.

* Respondents who reported consuming five or more alcoholic drinks (men) or four or more alcoholic drinks (women) on at least one occasion in the past 30 days.

† State prevalence estimates are divided into tertiles.

The higher values for the 90th percentiles for frequency ( 9.5 occasions in the past 30 days) and intensity ( 11.5 drinks on an occasion) indicate that a small proportion of adults binge drink very frequently, consume large quantities of alcohol, or both, which is consistent with previous findings (4).

Binge drinking prevalence decreased from $18.9 \%$ in 2011 to $18.0 \%$ in 2017 (5). This report found binge drinking prevalence was $17.4 \%$ in 2018 , indicating that binge drinking remained common. Alcohol consumption patterns might have since changed, including during the COVID-19 pandemic. Collectively, all three measures (prevalence, frequency, and intensity) address a complex pattern of binge drinking. For example, lower education and income levels were associated with lower binge drinking prevalence, but among adults who reported binge drinking, those with less than a high school diploma reported higher frequency and intensity than did college graduates. Similarly, adults in the lowest income level binge drank more frequently than did adults in the highest income level. The finding that the prevalence of binge drinking was lower in the most rural counties than in the most urban counties is consistent with earlier reports (G). However, adults in the most rural counties who binge drank did so more frequently and at higher intensity than did adults in the most urban counties. The prevalence of binge drinking in Mississippi and in West Virginia was lower than in the United States overall, but Mississippi had the highest median frequency and West Virginia had the highest median intensity of binge drinking among all states.

\section{Summary}

What is already known about this topic?

Excessive alcohol use has contributed to declines in life expectancy. Binge drinking is a common and costly pattern of excessive alcohol use.

What is added by this report?

During 2018, one in six U.S. adults reported binge drinking during the past 30 days. Among those who binge drank, 25\% did so at least weekly, on average, and $25 \%$ consumed at least eight drinks during a binge occasion. Some sociodemographic groups and states with low binge drinking prevalence reported large quantities of alcohol consumed during binge occasions.

What are the implications for public health practice?

An effective population health approach including regulating alcohol sales, increasing alcohol taxes, and alcohol screening and brief counseling by clinicians can help reduce binge drinking.

Excessive alcohol use is associated with increasing mortality from alcoholic liver disease, which has contributed to recently observed declines in U.S. life expectancy, notably among men, young and middle-aged adults, and persons with less than a high school education and limited income living in rural areas (7). The results of this study highlight the importance of reducing binge drinking, particularly among groups who are disproportionately affected.

The findings in this report are subject to at least three limitations. First, the BRFSS response rate indicates the potential for selection bias to the extent that survey respondents differ from nonrespondents. Second, responses are self-reported and subject to recall, social desirability, and nonresponse biases, which could vary across states and groups, and lead to underestimates of binge drinking (8). A study comparing BRFSS estimates to alcohol sales data found that although they were consistently correlated, survey data substantially underestimated consumption (9). Finally, binge drinking intensity based on the largest number of drinks reported on any occasion in the past 30 days might overestimate intensity. A previous analysis found that among demographic groups, this measure was 0.1-1.2 drinks higher than the reported number of drinks consumed during the most recent binge, but the two measures were strongly correlated (3). However, they were not correlated among adults without a high school diploma; in 2018, intensity by education level was highest among this group.

A population health approach has been shown to reduce excessive drinking, including binge drinking. The Community Preventive Services Task Force recommends the following strategies to reduce excessive drinking: increasing alcohol taxes, limiting hours and days of alcohol sales, and regulating alcohol outlet density. Fewer than one half of adults who report binge drinking to a health care provider during a medical checkup 
TABLE 2. Binge drinking frequency and intensity among adults aged $\geq 18$ years who binge drank in the past 30 days, by selected characteristics and state - Behavioral Risk Factor Surveillance System, United States, 2018

\begin{tabular}{|c|c|c|c|c|c|c|}
\hline \multirow[b]{4}{*}{ Characteristic } & \multicolumn{6}{|c|}{ Adults who binge drank in the past 30 days } \\
\hline & \multirow{2}{*}{\multicolumn{3}{|c|}{$\begin{array}{c}\text { Binge drinking }^{\dagger} \text { frequency } \\
\text { No. of }(n=54,045) \\
\text { occasions }{ }^{* *}(95 \% \mathrm{Cl})\end{array}$}} & \multicolumn{3}{|c|}{ Binge drinking ${ }^{\dagger}$ intensity $(n=50,527)$} \\
\hline & & & & & o. of drinks** $(950$ & \\
\hline & Median & 75th percentile & 90th percentile & Median & 75th percentile & 90th percentile \\
\hline Overall, unadjusted & $1.7(1.7-1.7)$ & $4.0(3.9-4.1)$ & $9.5(9.4-9.7)$ & $5.5(5.5-5.5)$ & $7.7(7.6-7.8)$ & $11.5(11.4-11.6)$ \\
\hline $\begin{array}{l}\text { Age group, yrs } \\
18-24 \\
25-34 \\
35-44 \\
45-64 \\
\geq 65\end{array}$ & $\begin{array}{l}1.7(1.6-1.8) \\
1.6(1.5-1.7) \\
1.7(1.6-1.8) \\
1.8(1.7-1.9) \\
1.8(1.6-1.9)\end{array}$ & $\begin{array}{l}4.1(3.9-4.4) \\
3.8(3.6-3.9) \\
3.9(3.8-4.1) \\
4.3(4.0-4.4) \\
4.4(4.1-4.7)\end{array}$ & $\begin{array}{r}9.1(7.7-9.4) \\
9.1(7.8-9.4) \\
9.7(9.4-11.1) \\
10.0(9.7-11.9) \\
14.2(9.9-18.5)\end{array}$ & $\begin{array}{l}5.9(5.8-6.0) \\
5.7(5.7-5.8) \\
5.5(5.4-5.6) \\
5.3(5.2-5.3) \\
5.0(4.9-5.0)\end{array}$ & $\begin{array}{l}9.2(8.9-9.3) \\
8.9(8.2-9.1) \\
7.6(7.4-7.8) \\
7.0(6.7-7.2) \\
5.8(5.7-5.8)\end{array}$ & $\begin{array}{r}13.6(12.0-14.3) \\
11.9(11.7-12.3) \\
11.4(11.2-11.6) \\
11.1(9.9-11.3) \\
7.9(7.4-9.1)\end{array}$ \\
\hline $\begin{array}{l}\text { Sex } \\
\text { Men } \\
\text { Women }\end{array}$ & $\begin{array}{l}1.9(1.8-1.9) \\
1.4(1.3-1.5)\end{array}$ & $\begin{array}{l}4.6(4.4-4.7) \\
3.3(3.2-3.4)\end{array}$ & $\begin{array}{r}11.4(10.0-12.0) \\
6.8(6.2-7.3)\end{array}$ & $\begin{array}{l}5.9(5.9-6.0) \\
4.5(4.5-4.5)\end{array}$ & $\begin{array}{l}9.3(9.2-9.4) \\
5.6(5.5-5.7)\end{array}$ & $\begin{array}{r}12.7(12.0-13.8) \\
7.9(7.7-8.6)\end{array}$ \\
\hline $\begin{array}{l}\text { Education level } \\
\text { Less than high school graduate } \\
\text { High school graduate or equivalent } \\
\text { Some college } \\
\text { College graduate }\end{array}$ & $\begin{array}{l}2.3(2.0-2.6) \\
1.9(1.8-2.0) \\
1.7(1.6-1.8) \\
1.4(1.3-1.4)\end{array}$ & $\begin{array}{l}4.9(4.5-5.8) \\
4.6(4.4-4.8) \\
4.0(3.9-4.2) \\
3.3(3.1-3.4)\end{array}$ & $\begin{array}{r}19.1(13.6-24.9) \\
11.6(10.0-13.9) \\
9.5(9.2-9.7) \\
6.9(6.2-7.3)\end{array}$ & $\begin{array}{l}5.8(5.7-5.9) \\
5.7(5.6-5.7) \\
5.5(5.5-5.6) \\
5.2(5.2-5.3)\end{array}$ & $\begin{array}{r}9.7(9.3-10.0) \\
9.0(8.4-9.2) \\
7.7(7.5-7.8) \\
6.8(6.6-6.9)\end{array}$ & $\begin{array}{r}14.8(14.1-17.2) \\
11.9(11.8-12.9) \\
11.4(11.2-11.5) \\
9.6(9.5-9.7)\end{array}$ \\
\hline $\begin{array}{l}\text { Race/Ethnicity } \\
\text { White, non-Hispanic } \\
\text { Black, non-Hispanic } \\
\text { Hispanic } \\
\text { Al/AN, non-Hispanic } \\
\text { A/PI, non-Hispanic } \\
\text { Other, non-Hispanic }\end{array}$ & $\begin{array}{l}1.7(1.7-1.8) \\
1.8(1.7-2.0) \\
1.6(1.5-1.7) \\
2.2(1.8-2.9) \\
1.4(1.2-1.6) \\
1.9(1.7-2.2)\end{array}$ & $\begin{array}{l}4.1(4.0-4.2) \\
4.2(3.8-4.6) \\
3.7(3.5-3.9) \\
5.5(4.4-7.2) \\
3.3(2.7-3.9) \\
4.7(3.9-5.7)\end{array}$ & $\begin{array}{r}9.7(9.5-9.8) \\
9.7(8.4-12.3) \\
7.7(7.1-9.3) \\
18.8(14.1-25.0) \\
7.2(5.1-9.4) \\
11.4(9.5-14.4)\end{array}$ & $\begin{array}{l}5.5(5.5-5.6) \\
5.1(5.0-5.3) \\
5.7(5.6-5.8) \\
5.7(5.4-6.0) \\
5.3(5.1-5.5) \\
5.7(5.5-5.8)\end{array}$ & $\begin{array}{l}7.7(7.6-7.8) \\
6.3(6.0-6.7) \\
8.9(7.8-9.2) \\
9.1(7.4-9.7) \\
6.8(6.2-7.4) \\
8.3(7.7-9.3)\end{array}$ & $\begin{array}{r}11.5(11.4-11.6) \\
9.7(9.3-11.1) \\
11.8(11.5-12.0) \\
13.0(11.5-14.7) \\
9.9(9.4-11.3) \\
12.7(11.5-14.7)\end{array}$ \\
\hline $\begin{array}{l}\text { Annual household income } \\
<\$ 25,000 \\
\$ 25,000-\$ 49,999 \\
\$ 50,000-\$ 74,999 \\
\geq \$ 75,000\end{array}$ & $\begin{array}{l}1.9(1.8-2.0) \\
1.7(1.6-1.8) \\
1.8(1.7-1.9) \\
1.6(1.5-1.7)\end{array}$ & $\begin{array}{l}4.5(4.2-4.7) \\
4.0(3.8-4.3) \\
4.2(3.9-4.5) \\
3.8(3.7-3.9)\end{array}$ & $\begin{array}{r}14.2(11.4-14.8) \\
9.7(9.3-10.0) \\
9.5(9.1-9.9) \\
9.1(7.8-9.3)\end{array}$ & $\begin{array}{l}5.5(5.5-5.6) \\
5.5(5.5-5.6) \\
5.6(5.6-5.7) \\
5.5(5.4-5.5)\end{array}$ & $\begin{array}{l}8.0(7.7-9.0) \\
7.8(7.5-8.1) \\
8.0(7.8-9.0) \\
7.5(7.3-7.6)\end{array}$ & $\begin{array}{l}11.8(11.6-12.0) \\
11.6(11.4-11.9) \\
11.7(11.5-11.9) \\
11.2(11.1-11.4)\end{array}$ \\
\hline $\begin{array}{l}\text { Marital status } \\
\text { Married }^{\dagger \dagger} \\
\text { Divorced/Separated/ Widowed } \\
\text { Never married }\end{array}$ & $\begin{array}{l}1.6(1.5-1.6) \\
1.9(1.8-2.0) \\
1.8(1.7-1.9)\end{array}$ & $\begin{array}{l}3.7(3.6-3.8) \\
4.8(4.5-5.2) \\
4.2(4.0-4.4)\end{array}$ & $\begin{array}{r}9.2(8.0-9.4) \\
18.2(14.5-19.9) \\
9.3(9.0-9.5)\end{array}$ & $\begin{array}{l}5.4(5.3-5.4) \\
5.4(5.3-5.5) \\
5.8(5.7-5.9)\end{array}$ & $\begin{array}{l}7.2(7.1-7.3) \\
7.6(7.3-7.8) \\
9.1(8.8-9.2)\end{array}$ & $\begin{array}{l}11.0(10.0-11.2) \\
11.6(11.4-11.9) \\
12.0(11.8-12.7)\end{array}$ \\
\hline $\begin{array}{l}\text { Veteran status } \\
\text { Veteran } \\
\text { Nonveteran }\end{array}$ & $\begin{array}{l}1.9(1.7-2.0) \\
1.7(1.6-1.7)\end{array}$ & $\begin{array}{l}4.6(4.2-4.9) \\
4.0(3.9-4.1)\end{array}$ & $\begin{array}{r}14.3(10.9-15.4) \\
9.4(9.2-9.6)\end{array}$ & $\begin{array}{l}5.7(5.6-5.8) \\
5.5(5.4-5.5)\end{array}$ & $\begin{array}{l}7.9(7.6-9.0) \\
7.7(7.6-7.8)\end{array}$ & $\begin{array}{l}11.7(11.4-11.9) \\
11.5(11.4-11.6)\end{array}$ \\
\hline $\begin{array}{l}\text { County urban-rural status } \\
\text { L } \\
\text { Large central metro } \\
\text { Large fringe metro } \\
\text { Medium metro } \\
\text { Small metro } \\
\text { Micropolitan } \\
\text { Noncore }\end{array}$ & $\begin{array}{l}1.6(1.6-1.7) \\
1.6(1.5-1.7) \\
1.7(1.7-1.8) \\
1.9(1.8-2.0) \\
1.8(1.7-1.9) \\
1.9(1.8-2.1)\end{array}$ & $\begin{array}{l}3.9(3.7-4.0) \\
3.8(3.6-4.0) \\
4.2(4.0-4.4) \\
4.4(4.1-4.6) \\
4.5(4.2-4.8) \\
4.5(4.1-4.8)\end{array}$ & $\begin{array}{r}9.2(8.0-9.6) \\
9.0(7.7-9.4) \\
9.7(9.4-10.0) \\
9.9(9.4-11.9) \\
11.5(9.8-14.1) \\
12.0(10.0-14.3)\end{array}$ & $\begin{array}{l}5.5(5.4-5.5) \\
5.4(5.4-5.5) \\
5.5(5.5-5.6) \\
5.6(5.6-5.7) \\
5.6(5.5-5.7) \\
5.7(5.6-5.8)\end{array}$ & $\begin{array}{l}7.5(7.3-7.7) \\
7.5(7.3-7.7) \\
7.7(7.5-7.9) \\
9.0(7.9-9.2) \\
8.3(7.8-9.1) \\
9.1(8.3-9.3)\end{array}$ & $\begin{array}{l}11.3(11.0-11.5) \\
11.3(11.1-11.5) \\
11.5(11.3-11.7) \\
11.8(11.5-12.0) \\
11.9(11.7-14.1) \\
12.1(11.8-14.3)\end{array}$ \\
\hline $\begin{array}{l}\text { U.S. Census region } 9 \text { १ी } \\
\text { Northeast } \\
\text { Midwest } \\
\text { South } \\
\text { West }\end{array}$ & $\begin{array}{l}1.6(1.5-1.7) \\
1.8(1.7-1.8) \\
1.8(1.7-1.8) \\
1.6(1.5-1.7)\end{array}$ & $\begin{array}{l}3.7(3.6-3.9) \\
4.2(4.0-4.4) \\
4.3(4.1-4.6) \\
3.8(3.6-3.9)\end{array}$ & $\begin{array}{r}7.7(7.3-9.1) \\
9.7(9.5-10.0) \\
10.1(9.7-11.8) \\
9.1(7.8-9.4)\end{array}$ & $\begin{array}{l}5.4(5.3-5.4) \\
5.6(5.5-5.7) \\
5.5(5.5-5.6) \\
5.5(5.5-5.6)\end{array}$ & $\begin{array}{l}7.3(7.1-7.5) \\
8.0(7.9-8.7) \\
7.7(7.5-7.9) \\
7.7(7.5-7.8)\end{array}$ & $\begin{array}{r}11.0(9.9-11.3) \\
11.8(11.6-11.9) \\
11.5(11.4-11.7) \\
11.4(11.2-11.6)\end{array}$ \\
\hline $\begin{array}{l}\text { State } \\
\text { Alabama } \\
\text { Alaska } \\
\text { Arizona } \\
\text { Arkansas } \\
\text { California } \\
\text { Colorado } \\
\text { Connecticut }\end{array}$ & $\begin{array}{l}1.7(1.5-1.9) \\
1.9(1.4-2.7) \\
1.5(1.3-1.7) \\
1.9(1.6-2.3) \\
1.6(1.4-1.7) \\
1.6(1.4-1.7) \\
1.6(1.4-1.7)\end{array}$ & $\begin{array}{l}4.0(3.4-4.6) \\
4.4(3.7-5.1) \\
3.8(3.2-4.3) \\
4.5(3.8-5.5) \\
3.7(3.4-3.9) \\
3.7(3.4-4.0) \\
3.5(3.1-3.8)\end{array}$ & $\begin{array}{r}8.4(6.7-9.7) \\
9.6(5.8-14.1) \\
9.2(7.2-9.8) \\
11.2(9.3-14.6) \\
7.9(7.2-9.4) \\
9.1(7.3-9.6) \\
7.8(5.9-9.5)\end{array}$ & $\begin{array}{l}5.5(5.3-5.6) \\
5.4(5.2-5.6) \\
5.5(5.4-5.7) \\
5.6(5.4-5.8) \\
5.5(5.4-5.6) \\
5.6(5.5-5.7) \\
5.2(5.1-5.3)\end{array}$ & $\begin{array}{l}7.2(6.5-8.0) \\
7.1(6.0-7.6) \\
7.9(7.3-9.1) \\
9.2(7.4-9.7) \\
7.7(7.4-8.2) \\
7.6(7.3-7.8) \\
6.5(6.0-6.9)\end{array}$ & $\begin{array}{r}10.8(9.8-11.9) \\
9.6(7.9-11.4) \\
11.3(9.9-11.8) \\
11.8(11.3-14.0) \\
11.5(11.2-11.8) \\
11.4(9.9-11.8) \\
9.3(8.0-9.8)\end{array}$ \\
\hline
\end{tabular}

See table footnotes on the next page. 
TABLE 2. (Continued) Binge drinking frequency and intensity among adults aged $\geq 18$ years who binge drank in the past 30 days, by selected characteristics and state - Behavioral Risk Factor Surveillance System, United States, ${ }^{*} 2018$

\begin{tabular}{|c|c|c|c|c|c|c|}
\hline \multirow[b]{4}{*}{ Characteristic } & \multicolumn{6}{|c|}{ Adults who binge drank in the past 30 days } \\
\hline & \multirow{2}{*}{\multicolumn{3}{|c|}{$\begin{array}{c}\text { Binge drinking }^{\dagger} \text { frequency }^{\S}(n=54,045) \\
\text { No. of occasions }{ }^{* *}(95 \% \mathrm{Cl})\end{array}$}} & \multirow{2}{*}{\multicolumn{3}{|c|}{$\begin{array}{c}\text { Binge drinking }{ }^{\dagger} \text { intensity }{ }^{\uparrow}(n=50,527) \\
\text { No. of drinks** }(95 \% \mathrm{Cl})\end{array}$}} \\
\hline & & & & & & \\
\hline & Median & $75^{\text {th }}$ percentile & $90^{\text {th }}$ percentile & Median & $75^{\text {th }}$ percentile & $90^{\text {th }}$ percentile \\
\hline Delaware & $1.7(1.5-2.0)$ & $4.4(3.8-5.2)$ & $11.3(8.4-14.6)$ & $5.3(5.0-5.5)$ & $7.3(6.6-7.8)$ & $11.3(9.6-11.9)$ \\
\hline District of Columbia & $1.5(1.3-1.7)$ & $3.4(2.9-4.0)$ & $6.9(5.5-7.9)$ & $5.2(5.0-5.4)$ & $6.7(6.0-7.2)$ & $9.4(8.1-9.8)$ \\
\hline Florida & $1.8(1.6-2.0)$ & $4.9(4.1-5.8)$ & $14.2(9.7-19.7)$ & $5.5(5.3-5.6)$ & $7.3(6.9-7.7)$ & $11.1(9.7-11.7)$ \\
\hline Georgia & $1.6(1.5-1.8)$ & $4.1(3.7-4.5)$ & $9.7(8.3-13.9)$ & $5.3(5.1-5.5)$ & $7.5(7.1-7.9)$ & $11.5(11.0-11.8)$ \\
\hline Hawaii & $1.9(1.7-2.2)$ & $4.3(3.9-4.7)$ & $11.1(8.8-14.3)$ & $5.7(5.5-5.8)$ & $9.2(7.9-9.6)$ & $13.3(11.5-14.8)$ \\
\hline Idaho & $2.0(1.7-2.5)$ & $4.5(3.9-5.3)$ & $10.5(8.0-17.9)$ & $5.8(5.6-6.1)$ & $9.1(7.7-9.7)$ & $13.1(11.4-14.4)$ \\
\hline Illinois & $1.9(1.7-2.1)$ & $4.4(3.9-4.8)$ & $9.7(7.9-11.9)$ & $5.7(5.5-5.8)$ & $9.1(7.8-9.5)$ & $14.0(11.7-14.6)$ \\
\hline Indiana & $1.7(1.5-1.9)$ & $4.2(3.7-4.8)$ & $9.9(9.2-14.5)$ & $5.6(5.4-5.8)$ & $9.1(7.8-9.6)$ & $14.1(11.7-15.5)$ \\
\hline lowa & $1.9(1.8-2.0)$ & $4.3(4.0-4.5)$ & $9.5(9.1-9.9)$ & $5.8(5.7-5.9)$ & $9.2(8.8-9.4)$ & $11.9(11.7-12.7)$ \\
\hline Kansas & $1.6(1.4-1.7)$ & $3.8(3.5-4.1)$ & $8.6(7.1-9.8)$ & $5.6(5.5-5.7)$ & $7.8(7.5-9.0)$ & $11.5(11.1-11.9)$ \\
\hline Kentucky & $1.9(1.7-2.4)$ & $5.0(4.2-6.9)$ & $10.2(9.6-14.4)$ & $5.7(5.5-5.9)$ & $8.8(7.5-9.4)$ & $13.7(11.4-14.8)$ \\
\hline Louisiana & $2.0(1.7-2.4)$ & $4.7(4.2-5.5)$ & $9.9(9.1-14.1)$ & $5.5(5.3-5.6)$ & $7.4(6.7-8.0)$ & $11.3(9.8-11.9)$ \\
\hline Maine & $1.8(1.6-2.0)$ & $4.1(3.6-4.7)$ & $9.3(7.3-11.4)$ & $5.4(5.2-5.5)$ & $7.1(6.5-7.8)$ & $11.2(9.6-11.6)$ \\
\hline Maryland & $1.6(1.4-1.8)$ & $3.7(3.4-4.0)$ & $7.9(6.7-9.7)$ & $5.3(5.2-5.5)$ & $7.0(6.6-7.3)$ & $10.0(9.6-11.5)$ \\
\hline Massachusetts & $1.5(1.4-1.7)$ & $3.6(3.2-3.9)$ & $7.0(5.4-7.9)$ & $5.4(5.2-5.5)$ & $7.0(6.5-7.6)$ & $11.2(9.8-11.6)$ \\
\hline Michigan & $1.8(1.6-1.9)$ & $4.3(3.9-4.7)$ & $10.0(9.4-14.0)$ & $5.6(5.4-5.7)$ & $7.9(7.6-9.0)$ & $11.7(11.2-14.0)$ \\
\hline Minnesota & $1.5(1.4-1.6)$ & $3.7(3.5-3.9)$ & $9.1(7.4-9.4)$ & $5.5(5.4-5.6)$ & $7.7(7.5-7.9)$ & $11.3(11.0-11.6)$ \\
\hline Mississippi & $2.1(1.8-2.7)$ & $5.4(4.1-8.8)$ & $14.8(10.0-22.1)$ & $5.7(5.5-5.9)$ & $9.4(7.8-10.3)$ & $14.6(11.3-17.4)$ \\
\hline Missouri & $1.8(1.6-2.2)$ & $4.8(4.1-6.1)$ & $14.0(9.5-14.9)$ & $5.5(5.3-5.7)$ & $7.7(7.1-9.0)$ & $11.7(11.2-12.5)$ \\
\hline Montana & $1.6(1.4-1.8)$ & $4.0(3.5-4.5)$ & $9.1(6.9-14.3)$ & $5.4(5.3-5.6)$ & $7.3(6.8-7.8)$ & $10.4(9.6-11.5)$ \\
\hline Nebraska & $1.7(1.6-1.8)$ & $3.9(3.6-4.3)$ & $9.1(7.5-9.7)$ & $5.6(5.5-5.7)$ & $8.0(7.6-9.1)$ & $11.4(11.1-11.7)$ \\
\hline Nevada & $1.6(1.3-1.9)$ & $3.7(2.9-4.5)$ & $7.4(5.6-9.3)$ & $5.5(5.2-5.7)$ & $7.8(6.7-9.3)$ & $11.2(9.8-14.1)$ \\
\hline New Hampshire & $1.5(1.3-1.8)$ & $4.0(3.4-4.7)$ & $9.9(7.7-13.2)$ & $5.5(5.3-5.7)$ & $7.6(6.9-8.3)$ & $11.2(9.5-11.7)$ \\
\hline New Jersey & —*** & $3.5(2.9-3.9)$ & $7.2(4.7-9.6)$ & $5.2(4.9-5.6)$ & $7.3(6.3-9.1)$ & $9.7(9.4-9.9)$ \\
\hline New Mexico & $1.8(1.5-2.1)$ & $4.5(4.0-5.2)$ & $11.4(9.5-17.8)$ & $5.5(5.3-5.7)$ & $7.9(7.2-9.4)$ & $13.1(11.5-18.3)$ \\
\hline New York & $1.6(1.5-1.7)$ & $3.7(3.4-4.0)$ & $7.7(6.9-9.2)$ & $5.4(5.3-5.5)$ & $7.1(6.8-7.4)$ & $11.0(9.8-11.4)$ \\
\hline North Carolina & $1.8(1.5-2.2)$ & $4.6(3.9-5.5)$ & $10.7(7.7-14.6)$ & $5.4(5.3-5.6)$ & $7.0(6.4-7.7)$ & $10.5(9.7-11.3)$ \\
\hline North Dakota & $1.8(1.5-2.0)$ & $4.2(3.7-4.7)$ & $8.2(6.9-9.9)$ & $5.9(5.7-6.3)$ & $9.4(9.1-9.7)$ & $14.1(11.7-14.6)$ \\
\hline Ohio & $1.9(1.7-2.2)$ & $4.4(3.9-4.9)$ & $12.0(9.5-18.6)$ & $5.7(5.5-5.8)$ & $8.2(7.7-9.2)$ & $11.8(11.3-13.6)$ \\
\hline Oklahoma & $1.5(1.2-1.7)$ & $3.8(3.0-4.5)$ & $9.3(7.3-16.0)$ & $5.6(5.4-5.8)$ & $7.9(7.2-9.3)$ & $11.5(9.9-12.0)$ \\
\hline Oregon & $1.7(1.5-1.9)$ & $4.1(3.6-4.5)$ & $9.6(8.3-13.2)$ & $5.5(5.4-5.6)$ & $7.4(6.9-7.8)$ & $9.9(9.6-11.4)$ \\
\hline Pennsylvania & $1.8(1.5-2.0)$ & $3.9(3.6-4.4)$ & $7.9(7.0-10.1)$ & $5.5(5.3-5.6)$ & $7.9(7.4-9.1)$ & $11.5(10.0-12.7)$ \\
\hline Rhode Island & $1.6(1.4-1.8)$ & $3.8(3.1-4.4)$ & $8.5(6.5-9.9)$ & $5.4(5.1-5.6)$ & $7.2(6.7-7.7)$ & $9.7(9.2-10.5)$ \\
\hline South Carolina & $1.9(1.7-2.2)$ & $4.6(4.1-5.2)$ & $11.4(9.5-14.9)$ & $5.7(5.5-5.8)$ & $8.4(7.7-9.3)$ & $11.7(11.2-12.0)$ \\
\hline South Dakota & $1.7(1.4-2.1)$ & $4.0(3.5-4.6)$ & $7.8(5.9-9.3)$ & $5.7(5.5-5.9)$ & $7.9(7.4-9.2)$ & $11.6(9.9-12.8)$ \\
\hline Tennessee & $1.7(1.4-2.1)$ & $4.4(3.6-5.2)$ & $9.2(6.8-12.2)$ & $5.5(5.3-5.7)$ & $7.6(6.9-9.1)$ & $11.8(11.2-14.4)$ \\
\hline Texas & $1.8(1.5-2.0)$ & $4.1(3.6-4.9)$ & $9.9(7.6-14.0)$ & $5.6(5.4-5.8)$ & $9.0(7.6-9.5)$ & $11.7(11.2-14.2)$ \\
\hline Utah & $1.7(1.6-1.9)$ & $4.4(4.0-4.8)$ & $10.2(8.9-14.4)$ & $5.6(5.4-5.7)$ & $7.8(7.4-8.7)$ & $11.4(10.3-14.1)$ \\
\hline Vermont & $1.9(1.6-2.3)$ & $4.3(3.7-5.1)$ & $9.5(8.1-12.3)$ & $5.5(5.2-5.7)$ & $7.5(7.0-8.0)$ & $10.6(9.8-11.6)$ \\
\hline Virginia & $1.6(1.5-1.8)$ & $3.7(3.4-4.0)$ & $9.8(9.1-14.0)$ & $5.5(5.3-5.6)$ & $7.2(6.8-7.7)$ & $11.3(10.0-11.6)$ \\
\hline Washington & $1.5(1.4-1.7)$ & $3.6(3.3-3.9)$ & $9.2(7.0-9.8)$ & $5.3(5.1-5.4)$ & $6.8(6.3-7.2)$ & $9.9(9.5-11.3)$ \\
\hline West Virginia & $1.8(1.5-2.4)$ & $5.1(4.3-7.1)$ & $15.2(11.5-20.8)$ & $6.4(5.9-7.2)$ & $9.8(9.2-11.3)$ & $14.6(12.0-17.5)$ \\
\hline Wisconsin & $1.7(1.5-1.9)$ & $3.8(3.4-4.3)$ & $9.1(6.6-9.7)$ & $5.5(5.4-5.7)$ & $7.5(7.0-7.9)$ & $10.8(9.8-11.4)$ \\
\hline Wyoming & $1.5(1.3-1.7)$ & $4.1(3.5-4.6)$ & $10.4(8.3-14.3)$ & $5.5(5.4-5.7)$ & $7.5(6.8-8.8)$ & $11.6(11.0-12.5)$ \\
\hline
\end{tabular}

Abbreviations: $\mathrm{A} / \mathrm{PI}=$ Asian/Pacific Islander; $\mathrm{Al} / \mathrm{AN}=$ American Indian/Alaska Native; $\mathrm{Cl}=$ confidence interval.

* The study sample included adult respondents aged $\geq 18$ years from all 50 states and the District of Columbia with complete information on age, sex, and binge drinking and who reported binge drinking.

† Binge drinking was defined as consuming five or more drinks (men) or four or more drinks (women) on at least one occasion during the past 30 days.

$\S$ Number of binge drinking occasions in the past 30 days among adults who reported binge drinking.

"Largest number of drinks consumed on any occasion in the past 30 days among adults who reported binge drinking.

** Unadjusted weighted medians and percentiles were derived from SAS-callable SUDAAN "proc descript"; variance estimates were derived using the Taylor series linearization method.

t† Married includes unmarried cohabitating couples.

$\S \S$ Counties were classified using the 2013 National Center for Health Statistics Urban-Rural Classification Scheme for Counties. https://www.cdc.gov/nchs/data/ series/sr_02/sr02_166.pdf

१ศी Regions were based on U.S. Census Bureau definitions. https://www2.census.gov/geo/pdfs/maps-data/maps/reference/us_regdiv.pdf

*** Estimates are unreliable if the relative standard error is $>0.3$. 
are advised to reduce their drinking (10). Clinicians should follow the U.S. Preventive Services Task Force recommendation to screen all adults for alcohol misuse and provide brief intervention and referral to treatment as needed.

Corresponding author: Michele K. Bohm, mbohm@cdc.gov, 770-488-3928.

${ }^{1}$ Division of Population Health, National Center for Chronic Disease Prevention and Health Promotion, CDC; ${ }^{2}$ Division of HIV Prevention, National Center for HIV, Viral Hepatitis, STD, and TB Prevention, CDC.

All authors have completed and submitted the International Committee of Medical Journal Editors form for disclosure of potential conflicts of interest. No potential conflicts of interest were disclosed.

\section{References}

1. Esser MB, Sherk A, Liu Y, et al. Deaths and years of potential life lost from excessive alcohol use-United States, 2011-2015. MMWR Morb Mortal Wkly Rep 2020;69:1428-33. PMID:33001874 https://doi. org/10.15585/mmwr.mm6939a6

2. Sacks JJ, Gonzales KR, Bouchery EE, Tomedi LE, Brewer RD. 2010 national and state costs of excessive alcohol consumption. Am J Prev Med 2015;49:e73-9. PMID:26477807 https://doi.org/10.1016/j. amepre.2015.05.031

3. Esser MB, Kanny D, Brewer RD, Naimi TS. Binge drinking intensity: a comparison of two measures. Am J Prev Med 2012;42:625-9. PMID:22608381 https://doi.org/10.1016/j.amepre.2012.03.001
4. Esser MB, Sacks JJ, Sherk A, et al. Distribution of drinks consumed by U.S. adults by average daily alcohol consumption: a comparison of 2 nationwide surveys. Am J Prev Med 2020;59:669-77. PMID:32747177 https://doi.org/10.1016/j.amepre.2020.04.018

5. Kanny D, Naimi TS, Liu Y, Brewer RD. Trends in total binge drinks per adult who reported binge drinking-United States, 2011-2017. MMWR Morb Mortal Wkly Rep 2020;69:30-4. PMID:31945030 https://doi.org/10.15585/mmwr.mm6902a2

6. Matthews KA, Croft JB, Liu Y, et al. Health-related behaviors by urbanrural county classification-United States, 2013. MMWR Surveill Summ 2017;66(No. SS-5). PMID:28151923 https://doi.org/10.15585/ mmwr.ss6605a1

7. Woolf SH, Schoomaker H. Life expectancy and mortality rates in the United States, 1959-2017. JAMA 2019;322:1996-2016. PMID:31769830 https://doi.org/10.1001/jama.2019.16932

8. Stockwell T, Donath S, Cooper-Stanbury M, Chikritzhs T, Catalano P, Mateo C. Under-reporting of alcohol consumption in household surveys: a comparison of quantity-frequency, graduated-frequency and recent recall. Addiction 2004;99:1024-33. PMID:15265099 https://doi. org/10.1111/j.1360-0443.2004.00815.x

9. Nelson DE, Naimi TS, Brewer RD, Roeber J. US state alcohol sales compared to survey data, 1993-2006. Addiction 2010;105:1589-96. PMID:20626370 https://doi.org/10.1111/j.1360-0443.2010.03007.x

10. McKnight-Eily LR, Okoro CA, Turay K, Acero C, Hungerford D. Screening for alcohol use and brief counseling of adults-13 states and the District of Columbia, 2017. MMWR Morb Mortal Wkly Rep 2020;69:265-70. PMID:32163383 https://doi.org/10.15585/mmwr. mm6910a3 